TRANS · NÚM. II $\cdot 2007$

NOTAS $\cdot 247-257$

Se presentan algunas orientaciones prácticas de Coseriu en relación con la traducción de sus estudios a diversas lenguas, con especial atención al francés, así como algunas normas en cuanto a la utilización de determinados rasgos lingüisticos generales (evitar parónimos interlingüisticos distorsionadores) y estilisticos en la edición de sus obras. PALABRAS CLAVE: traducción/traductor, "falsos amigos", conceptos y términos (en alemán, francés y español) relativos a la lingüistica del hablar, a la semántica léxica, a la gramática.

\title{
[Pautas conceptuales, terminológicas y estilísticas para la traducción de mis obras: esbozo]
}

Some practical orientations are displayed in relation with the translation of Prof. Coseriu's works into several languages, specially into French. Furthermore some norms are given in order to avoid certain distorted uses (which do not belong to his "idiolect»).

KEYWORDS: translation/translator, "faux amis", terms (in German, French and Spanish) related with grammar, lexical semantics, linguistics of the performance.

EUGENIO COSERIU ( $(+)$

DISPUESTO PARA LA IMPRENTA POR JOSÉ POLO

Universidad Autónoma de Madrid 


\section{NOTA PREVIA}

o. La presentación al público lector de este memorando dúplice se realiza dentro del proyecto La obra científica de Eugenio Coseriu: ordenación, estudio y edición (Ministerio de Ciencia y Tecnología, Dirección General de Investigación, Subdirección General de Proyectos de Investigación, Plan Nacional de Investigación Científica, Desarrollo e Innovación Tecnológica: BFF2OO2-OI827).

I. Como puede observarse por la presencia de los corchetes en el propio título, se trata de una nominación creada por mí, no por el doctor Coseriu, para arropar dos guías pensadas por él como ayuda para los traductores de sus estudios y, sin duda, a la vista de experiencias no positivas al respecto o intentando prevenirlas (por supuesto, no es este el momento de detenerme en un análisis del hecho de las versiones de la obra coseriana no revisadas por él y en sus «peligros hermenéuticos» ante la falta de seguridad textual). Para que no pase inadvertido el carácter interno o «de andar por casa» de esos dos textos, no me ha importado redundar en tal idea haciendo que se sume lo denotado, y hasta lo connotado, de pautas y esbozo. He preferido juntar los dos instrumentos que enseguida transcribiré en una sola unidad como dos partes complementarias, pues ambos esquemas orientadores se dirigían al mismo objeto: contribuir a que la versión de sus trabajos ganase en calidad y, como consecuencia de ello, la penosa tarea de revisión por su parte se aligerase algo. Siempre que el profesor Coseriu laboraba con la traducción de algún texto suyo, lo hacía con enorme responsabilidad, vale decir, con un gran despliegue de esfuerzo cuando el texto sometido a su visto bueno no brillaba por el rasgo de excelencia.

2. La primera de las guías que voy a reproducir —existente en original mecanografiado, como la segunda, aparece nombrada como "Coseriu, Wichtigere Begriffe und Termini», esto es, Conceptos y términos más importantes [en su obra]. Pertenece, es obvio, a su etapa alemana, aunque no me es posible precisar el año (sospecho que es posterior a I985); lo veo mencionado, como «dactylographié», en la primera entrada bibliográfica (pág. 298) de los trabajos coserianos en el libro de Colette Laplace («Préface de Jean-Marie Zemb») Théorie du langage et théorie de la traduction. Les concepts-clefs de trois auteurs: Kade (Leipzig), Coseriu (Tübingen), Seleskovitch (Paris), Didier («Publié avec le concours du Centre de Recherches en Traductologie, Université de Paris III»), París, I994 (colección Traductologie, 8). Puesto que, en el conjunto de la obra de Coseriu, la primera lengua es el español - en el sentido, cuando menos, de que, a partir de lo publicado por él en este idioma, se han creado las pautas para la mayor parte de las ediciones en otras lenguas-, había pensado, en principio, cambiar el orden en esta primera guía (alemán, español, francés) y dejarlo así: español, francés, alemán (juntando las dos lenguas románicas y, por lo acabado de decir, colocando a la española como punto de referencia). Finalmente, he desistido de tal propósito para no «desestructurar», sin la posibilidad de consulta al doctor Coseriu, un esquema que nació con su propia «forma interior» germana. Lo que sí he hecho ha sido, manteniendo el orden de aparición de esas tres lenguas, no utilizar la cursiva para los términos de la situada en medio, la española, y de 
este modo atenuar el efecto de falta de legibilidad de la cursiva cuando se halla muy presente (en el original solo aparecía en cursiva la forma primera, alemana). Además, he colocado al principio lo que en el original se hallaba al final: las Abkürzungen o abreviaciones/abreviaturas/siglas. Igualmente, he añadido los datos bibliográficos que permitan reconocer en español los trabajos mencionados (la mayoría, en sus ediciones en lengua alemana), como también he dado las versiones al español de los ocasionales sintagmas explicativos en alemán presentes en la terminología que sirve de punto de partida para el espacio, digo, germanohablante. He presentado en forma completa algunas palabras que, por el contexto, aparecían abreviadas cuando se repetían (por ejemplo, B./Bedeutung). Por otro lado, esa armazón conceptualterminológica constituye, sin duda, un recordatorio de la necesidad de configurar en algún momento una especie de enciclopedia o diccionario, según, con el universo científico de nuestro autor (a lo que me he referido con mayor detalle en más de un trabajo sobre Coseriu).

3. El segundo memorando, Para el traductor, contiene normas de carácter general y otras (sección segunda del original; cuarta en el orden que he establecido para dejar al final la aplicación a una lengua determinada) proyectadas hacia el francés, lengua que entra más tarde que otras en el proceso de recopilación de estudios coserianos a través de diversos volúmenes. El que se encuentren, aquí y allá, y sin alejarse en el tiempo, traducciones de estudios de Coseriu poco cuidadas convertía en inaplazable la tarea de dar a conocer los textos de ahora, «instrumentales», sobre todo cuando el maestro no puede ayudarnos directamente en tan decisiva labor, uno de los pilares del carácter universal de su obra. He dejado tal cual el uso «coloquial» o genérico del infinitivo («dejar más espacio» frente a «déjese más espacio», etc.) como llamada de atención sobre el carácter sencillo de estas notas, probablemente o dictadas por el profesor Coseriu y escritas por otra persona o mecanografiadas por la aludida segunda persona a partir de un original manuscrito (de letra incluso rápida, por los errores mecanográficos que he corregido directamente sin interpolación alguna: un sino, de claro sentido condicional, que he convertido en si no, falta o sobra de alguna coma y algún otro hecho gráfico). Para evitar comenzar frase con palabras en minúscula (como hay que evitarlo, igualmente, si se inicia con un número arábigo...), aunque se trate de lo que yo denomino «metalenguaje de ilustración», he interpolado soportes del tipo La expresión, El sintagma, etc. Puede resultar pesado, pero lo he preferido, como mal menor, a romper el paralelismo establecido por el doctor Coseriu entre unas formas y otras, en su expresión neutra, a través de la niveladora «esencial» minúscula.

4. Quisiera, por último, añadir que me he permitido reajustar ortotipográficamente los dos frentes textuales, de manera que el tránsito de lo mecanográfico a lo tipográfico se realice con naturalidad y «eficacia visual» (he sustituido los «guiones iniciales» — que deberían haber sido, en todo caso, «rayas ordinales»—-, ante cada una de las indicaciones, por números arábigos; etc.). El haber preparado para su edición diversos escritos de Coseriu en vida de él con su beneplácito - también en los asuntos, no tan secundarios, de la presentación material- me hace mirar estas cuestiones distendidamente, con tranquilidad absoluta, dada la atención que siempre he puesto en todo lo que, bajo mi responsabilidad, va camino de convertirse en letra de molde, como 
podrá observarse, salvo estropicios ajenos a mi voluntad, en todo lo que vengo publicando en los últimos años.

PRIMERA PARTE

WICHTIGERE BEGRIFFE UND TERMINI/CONCEPTOS Y TÉRMINOS MÁS IMPORTANTES

\section{Abkürzungen/Siglas}

$L L=$ «Die Lage in der Linguistik» «La "situación” en la lingüística», I97I en inglés y 1973 en alemán; cap. XI en El hombre y su lenguaje, 1977].

$D E=$ «Determinación y entorno» [con el subtítulo de «Dos problemas de una lingüística del hablar»; 1955-1956; recogido en Teoría del lenguaje y lingüistica general, 1962].

$S u G=$ «Semantik und Grammatik» [1971; «Semántica y gramática»; cap. v en Gramática, semántica, universales, 1978].

$F B W=$ «Die funktionelle Betrachtung des Wortschatzes» [1976; «El estudio funcional del vocabulario»; cap. viI en GSU ].

$E S L=$ «Introduction à l'étude structurale du lexique» [1964 oralmente, I966 impreso; «Introducción al estudio estructural del léxico»; cap. II en Principios de semántica estructural, 1977].

$L o g=$ «Logicismo y antilogicismo en la gramática» [1956; recogido en TLLG].

$K G=$ «Über Leistung und Grenzen der kontrastiven Grammatik» [1970; «Alcances y límites de la gramática contrastiva»; cap. III en GSU].

$S D S=$ «Pour une sémantique diachronique structurale» [1964; «Para una semántica diacrónica estructural»; cap. I en PSE].

$S L=$ «Les structures lexématiques» [1968; «Las estructuras lexemáticas»; cap. IV en PSE].

$L S=$ «Lexikalische Solidaritäten» [1976; «Las solidaridades léxicas»; cap. III en PSE]. 
I

\section{ALLGEMEINE BEGRIFFE/CONCEPTOS GENERALES}

I. Sprache (wenn allgemein verwendet/empleado en general); lenguaje; langage (DE, LL).

Sprechen im Allgemeinen; hablar; parole en général.

Einzelsprache; lengua; langue (bzw./esto es, Sprachen/[las, diversas, determinadas] lenguas, im Plural; eine Sprache usw./una lengua dada, etc.).

«Text»; discurso («texto»); discours («texte»).

2. Inhalt; contenido; contenu (LL, SuG, FBW)

Bezeichnung; designación; désignation.

Bedeutung; significado; signifié (signification).

Sinn; sentido; sens.

3. Bedeutung; significado; signifié (SuG, FBW).

lexikalische Bedeutung; significado léxico; signifié lexical.

kategorielle Bedeutung; significado categorial; signifié catégoriel.

instrumentale Bedeutung; significado instrumental; signifié instrumental.

strukturelle oder syntaktische (innerstrukturelle) Bedeutung; significado estructural o sintáctico; signifié structural ou syntaxique.

ontische Bedeutung; significado óntico; signifié ontique.

4. Sprache/«Sachen» (außersprachliche Wirklichkeit); lenguaje/«cosas» (realidad extralingüística); langage/«choses» (réalité extralinguistique) (ESL).

5. primäre Sprache/Metasprache; lenguaje primario/metalenguaje; langage primaire/ métalangage (ESL).

6. universell/allgemein (empirisch allgemein); universal/general; universel/général (Log, KG).

Universalität, universalidad; universalité.

Allgemeinheit, generalidad; généralité.

7. historische Sprache; lengua histórica (idioma); langue historique (ESL).

8. (freie) Sprachtechnik, Technik der Rede/wiederholte Rede; técnica (libre), técnica del discurso/discurso repetido; technique (libre), technique du discours/discours répété (ESL).

9. Synchronie/Diachronie; sincronía/diacronía; synchronie/diachronie (ESL).

synchronisch/diachronisch; sincrónico/diacrónico; synchronique/diachronique. 
Io. Syntopie/Diatopie; sintopía/diatopía; syntopie/diatopie (ESL).

syntopisch/diatopisch; sintópico/diatópico; syntopique/diatopique.

II. Synstratie/Diastratie; sinstratía/diastratía; synstratie/diastratie (ESL).

synstratisch/diastratisch; sinstrático[yo, J. P., suelo emplear sinéstrático]/diastrático; synstratique/diastratatique.

I2. Symphasie/Diaphasie; sinfasía/diafasía; symphasie/diaphasie (ESL).

symphasisch/diaphasisch; sinfásico/diafásico; symphasique/diaphasique.

I3. Architektur der Sprache; arquitectura de la lengua [yo, J. P., suelo emplear «arquitectura del lenguaje»]; architecture de la langue (ESL).

Dialekt (Mundart); dialecto; dialecte.

Sprachniveau; nivel (de lengua); niveau (de langue).

Sprachstil; estilo de lengua; style de langue.

I4. funktionelle Sprache; lengua funcional; langue fonctionnelle (ESL).

Struktur; estructura; structure.

Paradigma/Syntagma; paradigma/sintagma; paradigme/syntagme (SL).

paradigmatische Struktur, paradigmatische Funktion; estructura paradigmática,

función paradigmática; structure paradigmatique, fonction paradigmatique.

syntagmatische Struktur, syntagmatische Funktion; estructura sintagmática, función

sintagmática; structure syntagmatique, fonction syntagmatique.

Ebenen der Sprachtechnik; planos de estructuración (de la técnica de una lengua); plans de structuration (ESL).

Sprachtypus; tipo lingüístico; type linguistique.

Sprachsystem; sistema (de la lengua); système (de la langue).

Sprachnorm; norma (de la lengua); norme (de la langue).

Rede; habla; parole.

II

FUNKTIONELLE BETRACHUNG DER SPRACHE (GRAMMATIK UND LEXIKOLOGIE) CONSIDERACIÓN FUNCIONAL DEL LENGUAJE

15. Prinzip der Funktionalität; principio de la funcionalidad; principe de la fonctionnalité (FBW).

einheitliche Bedeutung; significado unitario; signifié unitaire.

Kommutation; conmutación; conmutation.

(funktionelle) Einheit/Variante; unidad (funcional)/variante; unité (fonctionnelle)/ variante. 
16. Prinzip der Opposition; principio de la oposición; principe de l'opposition (FBW). unterscheidender Zug; rasgo distintivo; trait distinctif.

I7. Prinzip der Systematizität, principio de la sistematicidad; principe de la systématicité (FBW).

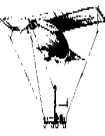

253

18. Prinzip der Neutralisation; principio de la neutralización; principe de la neutralisation (FBW). Neutralisation(Neutralisierung)/Synkretismus; neutralización/sincretismo; neutralisation/ syncrétisme (SDS). extensiv/intensiv; extensivo/intensivo; extensif/intensif. Archieinheit, Hypoeinheit; archiunidad, hipounidad; archiunité, bypounité.

III

\section{GRAMMATIK}

I9. Logizismus/Antilogizismus; logicismo/antilogicismo; logicisme/antilogicisme (LoG).

20. Morphemwörter/Kategoremwörter/Lexemwörter, morfemas, categoremas, lexemas; morphèmes/catégorèmes/lexèmes (SuG).

Redeteile (Worarten); categorías verbales (partes de la oración); catégories verbales (parties du discours).

2I. grammatische Schichten; estratos gramaticales; couches grammaticales (KG). minimale Einheit; unidad míníma; unité minimale.

Wort; palabra; mot.

Wortgruppe; grupo de palabras; groupe de mots.

Klausel; cláusula; clausule.

Satz; oración; phrase.

Text; texto; texte.

22. Eigenschaften der grammatischen Schichten; propiedades de los estratos gramaticales; propriétés des couches grammaticales (KG).

Superordinierung; superordinación; superordination.

Subordinierung; subordinación; subordination.

Koordinierung; coordinación; coordination.

Ersetzung; substitución [yo, J. P., prefiero la forma $\sin b$ ]; substitution.

23. Bereiche der Grammatik; dominios de la gramática; domaines de la grammaire(KG). konstitutionelle Grammatik; gramática constitucional; grammaire constitutionnelle. funktionelle Grammatik; gramática funcional; grammaire fonctionnelle. relationelle Grammatik; gramática relacional; grammaire relationnelle. 
24. Konfiguration; configuración (asociativa); configuration (associative) (SDS).

25. primäre Strukturen; estructuras primarias; structures primaires (SL, FBW).

Wortfeld; campo léxico; champ lexical.

Lexem; lexema; lexème.

Sem; sema; sème.

Archilexem; archilexema; archilexème.

Wortklasse; clase léxica; classe lexicale.

Klassem; clasema; classème.

26. sekundäre Strukturen; estructuras secundarias; structures secondaires (SL, FBW).

Modifikation (Modifizierung); modificación; modification.

Entwicklung; desarrollo; développment.

Komposition; composición; composition.

lexematische Komposition; composición lexemática; composition lexématique.

prolexematische Komposition; composición prolexemática; composition prolexématique.

27. lexikalisches Solidaritäten;solidaridades léxicas; solidarités lexicales (SL, LS).

Affinität; afinidad; affinité.

Selektion; selección; sélection.

Implikation; implicación; implication.

28. Determinierung; determinación; détermination.

V

LINGUISTIK DES SPRECHEN, LINGÜÍSTICA DE HABLAR, LINGUISTIQUE DE LA PAROLE (EN GÉNÉRAL) (DE)

Aktualisierung; actualización; actualisation.

Diskrimination; discriminación; discrimination.

Quantifizierung; cuantificación; quantification.

Selektion; selección; sélection.

Partikularisierung; particularización; particularisation.

Individuierung; individuación; individuation.

Individualisierung; individualización; individualisation.

Situierung; situación; situation.

Lokalisierung; localización; localisation.

Abgrenzung; delimitación; délimitation.

Explizierung; explicación; explication.

Spezialisierung; especialización; spécialisation.

Spezifizierung; especificación; spécification.

Identifizierung; identificación; identification. 
29. Umfeld; entorno; entourage.

Situation; situación; situation.

Region; región; région.

Zone; zona; zone.

Bereich; ámbito; milieu (objectif).

Umgebung; ambiente; ambiance.

Kontext, contexto; contexte.

einzelsprachlicher Kontext; contexto idiomático; contexte de langue.

Rede-Kontext, contexto verbal; contexte verbal.

Außer-Rede-Kontext; contexto extraverbal; contexte extraverbal.

Redeuniversum; universo de discurso; univers de discours.

\section{SEGUNDA PARTE}

PARA EL TRADUCTOR

$\mathrm{I}-\mathrm{I}$

I. Dejar más espacio entre los renglones.

2. Dejar margen.

3. No escribir nada entre las líneas.

4. Para los cambios en el orden de las palabras, emplear numeritos.

I-4. Si no, no hay espacio suficiente para las correcciones. De las últimas traducciones, algunas las he debido hacer copiar para poder corregirlas.

\section{$\mathrm{I}-2$}

5. No traducir nunca los ejemplos. Dejar[me] a mí el cuidado de adaptarlos o de encontrar ejemplos análogos. A veces las relaciones implicadas por las palabras traducidas son exactamente lo contrario de las de los ejemplos originales.

II

6. [La forma] mismo como adjetivo presenta siempre concordancia; así que: las cosas mismas, las cosas en sí mismas (no las cosas mismo).

7. No es bueno emplear general e independiente como adverbios. Decir generalmente, independientemente.

8. No hay que emplear un con esp. cierto de valor indefinido: en cierto nivel, no en un cierto nivel.

9. [La estructura] más bien que, en sentido negativo, se construye en español con el infinitivo: más bien que implicarlo (no más bien que no lo implica).

ı. [Las abreviaturas] fr., esp., it., etc., delante de los ejemplos se emplean sin artículo. 
Tratar de adaptar la traducción a la lengua y a las costumbres idiomáticas de los textos escritos originariamente en español. Por ejemplo...

II. Yo no empleo nunca constatar, sino, según los casos, comprobar, identificar, advertir, encontrar, etc.

I2. Empleo normalmente de este modo y sólo raramente de esta forma (además, no conviene emplear esta expresión en contextos en que ya se habla de formas [y aparece, por consiguiente, más de una vez forma]).

13. No emplear siempre incluso por aun, hasta.

I4. No emplear concretamente por precisamente, a saber, es decir, o sea.

15. En el sentido de sólo, nada más que, conviene emplear simplemente (no sencillamente).

\section{IV}

\section{[APLICACIÓN AL FRANCÉS]}

16. Ne...que es la manera francesa de decir sólo. No hay que traducir constantemente por no... más que, no... sino. Emplear, todas las veces que ello sea posible, simplemente sólo. También ce n'est que equivale simplemente a sólo.

I7. En francés se dice siempre le fait de+infinitivo porque el francés no tiene otra posibilidad. En español esto corresponde, en la mayoría de los casos, al infinitivo sustantivado. Así que: el hacer, el considerar, no el hecho de hacer, el hecho de considerar [estructuras posibles y normales en situaciones comunicativas distintas: mayor relieve, etcétera].

I8. A fr. on fait corresponde en español muy a menudo la primera persona del plural: no hay que traducir siempre por el impersonal. Y la equivalencia de fr. on + verbo + objeto es en español se + verbo + sujeto; así que: on peut employer $x$ — puede emplearse $x$; on fait des choses_ se hacen cosas. Evitar, de todos modos, el tipo uno hace.

19. En cambio, a la r. ${ }^{\mathrm{a}}$ persona del plural de[1] francés puede corresponder en español el impersonal con se: hay que elegir lo más corriente en español (por ej., nous avons vuse ha visto; comme nous l'avons dit__ como ya se ha dicho).

20. El francés que que encabeza una segunda oración subordinada representa a la conjunción subordinante de la primera y no se traduce.

2I. Para fr. ce qui est petit, ce qui est grand, etc., el español tiene lo pequeño, lo grande (no lo que es pequeño).

22. [La forma] aussi en el comienzo de la oración no significa también, sino por consiguiente, por lo tanto.

23. La equivalencia más corriente de il faut es hay que (o forma de tener que, deber), no es necesario.

24. [La estructura $]$ tout en + gerundio no tiene nada que ver con todo; significa aun+gerundio.

25. [El sintagma] à l'aide de corresponde normalmente a por medio de, mediante (no a con la ayuda de). 
26. [La estructura] en tant que corresponde a en cuanto o, simplemente, a como.

27. [El sintagma] de ce fait no significa de hecho, sino por ello, por lo mismo, de aqui que.

28. [El sintagma] avoir affaire significa normalmente ocuparse de, enfrentarse con, encontrar, hallarse frente a, tratarse de y sólo raramente tener que ver con.

29. [El verbo] croire es a menudo pensar (no creer).

30. [La expresión] s'arrêter à (un objeto de estudio) es atender a.

3I. [La estructura] c'est le cas corresponde a se da, se nota, se comprueba, encontramos; no emplear siempre es el caso.

32. [La expresión] tout au plus es a menudo a lo sumo (no emplear siempre todo lo más).

33. [La forma] or significa ahora bien, pero, no por tanto.

34. [La forma] partout no significa sobre todo, sino en todas partes.

35. [La expresión] en vue de no significa a la vista de, sino con la finalidad de.

36. [La expresión] un seul et même corresponde a un mismo.

37. [La expresión] si bien no significa si bien que, aunque, sino de manera que, asi que.

38. [La estructura] par rapport à es a menudo en comparación con, comparado con; otras veces, para.

39. [La expresión] en soi es a menudo como tal (no en sí).

40. [La forma] sinon es normalmente de otro modo.

4I. [La estructura] on est autorisé à corresponde a es lícito, se puede.

42. [La forma] une como adjetivo significa único, unitario, mismo (une langue une-una lengua única o una lengua unitaria, una misma lengua).

43. [La expresión] en tout cas como inciso corresponde a de todos modos.

44. [La estructura] aussi+ adjetivo es igualmente + adjetivo o tan ... como (no también); por ej., aussi nombreux_ igualmente numerosos (no también numerosos).

45. La equivalencia normal de italiques (lettres) es bastardilla [o, más corriente, cursiva]. 